\title{
A GENERAL EFFECTIVE THEORY FOR DENSE QUARK MATTER
}

\author{
P. T. REUTER, Q. WANG AND D. H. RISCHKE \\ Institute für Theoretische Physik, \\ Johann Wolfgang Goethe - Universität, \\ Robert-Mayer-Str. 10 , \\ D-60054 Frankfurt am Main, Germany
}

\begin{abstract}
A general effective action for quark matter at nonzero temperature and/or nonzero density is derived. Irrelevant quark modes are distinguished from relevant quark modes, and hard from soft gluon modes, by introducing two separate cut-offs in momentum space, one for quarks, $\Lambda_{q}$, and one for gluons, $\Lambda_{g}$. Irrelevant quark modes and hard gluon modes are then exactly integrated out in the functional integral representation of the QCD partition function. Depending on the specific choice for $\Lambda_{q}$ and $\Lambda_{g}$, the resulting effective action contains well-known effective actions for hot and/or dense quark matter, for instance the "Hard Thermal Loop" (HTL) or the "Hard Dense Loop" (HDL) action, as well as the high-density effective theory proposed by Hong and others.
\end{abstract}

\section{Introduction}

Sufficiently cold and dense quark matter is a color superconductor ${ }^{1}$. Due to asymptotic freedom, at asymptotically large quark chemical potential, $\mu \gg \Lambda_{\mathrm{QCD}}$, the strong coupling constant $g \ll 1$, and one can apply weakcoupling methods to compute the color-superconducting gap parameter. For $N_{f}$ massless quark flavors which participate in the screening of gluon fields, to subleading order in $g$ the result is $2,3,4,6,7$

$$
\phi=512 \pi^{4}\left(\frac{2}{N_{f} g^{2}}\right)^{5 / 2} \mu \exp \left(-\frac{3 \pi^{2}}{\sqrt{2} g}-\frac{\pi^{2}+4}{8}\right)[1+O(g)] .
$$

Sub-subleading corrections enter as $O(g)$ corrections to the prefactor of the exponential. For a reliable extrapolation of this result to quark chemical potentials of physical interest, $\mu \sim 500 \mathrm{MeV}$, it is mandatory to know the order of magnitude of these sub-subleading corrections. However, in full QCD their calculation appears prohibitively difficult. In order to proceed, note that in weak coupling, $g \ll 1$, there is an ordering of scales $\phi \sim$ 
$\mu \exp (-1 / g) \ll g \mu \ll \mu$. This ordering of scales implies that the modes near the Fermi surface, which participate in the formation of Cooper pairs and are therefore of primary relevance in the gap equation, can be considered to be independent of the detailed dynamics of the modes deep within the Fermi sea. This suggests that the most efficient way to compute properties such as the color-superconducting gap parameter is via an effective theory for quark modes near the Fermi surface. Such an effective theory has been originally proposed by Hong ${ }^{8,9}$ and was subsequently refined by others $10,11,12,13$.

In this paper, we derive a general effective theory for hot and/or dense quark matter from first principles (see also Ref. ${ }^{14}$ ). We introduce cut-offs in momentum space for quarks, $\Lambda_{q}$, and gluons, $\Lambda_{g}$. These cut-offs separate relevant from irrelevant quark modes and soft from hard gluon modes. We then explicitly integrate out irrelevant quark and hard gluon modes. We show that the standard HTL and HDL effective actions are contained in our general effective action for a certain choice of the quark and gluon cutoffs $\Lambda_{q}, \Lambda_{g}$. It can also be shown ${ }^{14}$ that the action of the high-density effective theory derived by Hong and others ${ }^{8,9,10,11,12,13}$ is a special case of our general effective action. We also outline the computation of the colorsuperconducting gap parameter with this effective action (for more details, see Ref. ${ }^{14}$ ).

Our units are $\hbar=c=k_{B}=1$. 4-vectors are denoted by $K^{\mu}=\left(k_{0}, \mathbf{k}\right)$, with $\mathbf{k}$ being a 3 -vector of modulus $|\mathbf{k}| \equiv k$. For the summation over Lorentz indices, we use the familiar Minkowski metric $g^{\mu \nu}=\operatorname{diag}(+,-,-,-)$, although we exclusively work in compact Euclidean space-time with volume $V / T$, where $V$ is the 3 -volume and $T$ the temperature of the system. Spacetime integrals are denoted as $\int_{0}^{1 / T} d \tau \int_{V} d^{3} \mathbf{x} \equiv \int_{X}$. Energy-momentum sums are denoted as $(T / V) \sum_{K} \equiv T \sum_{n}(1 / V) \sum_{\mathbf{k}}$. The sum over $n$ runs over the Matsubara frequencies, $\omega_{n}^{\mathrm{b}}=2 n \pi T$ for bosons and $\omega_{n}^{\mathrm{f}}=(2 n+1) \pi T$ for fermions. The 4-dimensional delta-function is conveniently defined as $\delta^{(4)}(X) \equiv \delta(\tau) \delta^{(3)}(\mathbf{x})$.

\section{Deriving the Effective Theory}

The partition function for QCD in the absence of external sources reads

$$
Z=\int[D A] \exp \left\{S_{A}[A]\right\} Z_{q}[A]
$$


Here the (gauge-fixed) gluon action is

$$
S_{A}[A]=\int_{X}\left[-\frac{1}{4} F_{a}^{\mu \nu}(X) F_{\mu \nu}^{a}(X)\right]+S_{\mathrm{gf}}[A]+S_{\mathrm{ghost}}[A],
$$

where $F_{\mu \nu}^{a}=\partial_{\mu} A_{\nu}^{a}-\partial_{\nu} A_{\mu}^{a}+g f^{a b c} A_{\mu}^{b} A_{\nu}^{c}$ is the gluon field strength tensor, $S_{\text {gf }}$ is the gauge-fixing part, and $S_{\text {ghost }}$ the ghost part of the action.

The partition function for quarks in the presence of gluon fields is

$$
Z_{q}[A]=\int[D \bar{\Psi}][D \Psi] \exp \left\{S_{q}[A, \bar{\Psi}, \Psi]\right\}
$$

where the quark action is

$S_{q}[A, \bar{\Psi}, \Psi]=\frac{1}{2} \int_{X, Y} \bar{\Psi}(X) G_{0}^{-1}(X, Y) \Psi(Y)+\frac{g}{2} \int_{X} \bar{\Psi}(X) \hat{\Gamma}_{a}^{\mu} A_{\mu}^{a}(X) \Psi(X)$.

Here,

$$
\Psi \equiv\left(\begin{array}{c}
\psi \\
\psi_{C}
\end{array}\right), \quad \bar{\Psi} \equiv\left(\bar{\psi}, \bar{\psi}_{C}\right),
$$

are Nambu-Gor'kov quark spinors, where $\psi_{C} \equiv C \bar{\psi}^{T}, \bar{\psi}_{C} \equiv \psi^{T} C$ are charge-conjugate quark spinors; $C \equiv i \gamma^{2} \gamma_{0}$ is the charge conjugation matrix. The free inverse quark propagator in the Nambu-Gor'kov basis is

$$
G_{0}^{-1}(X, Y) \equiv\left(\begin{array}{cc}
{\left[G_{0}^{+}\right]^{-1}(X, Y)} & 0 \\
0 & {\left[G_{0}^{-}\right]^{-1}(X, Y)}
\end{array}\right)
$$

with the free inverse propagator for quarks and charge-conjugate quarks

$$
\left[G_{0}^{ \pm}\right]^{-1}(X, Y) \equiv\left(i \not \partial_{X} \pm \mu \gamma_{0}-m\right) \delta^{(4)}(X-Y) .
$$

The quark-gluon vertex in the Nambu-Gor'kov basis is defined as

$$
\hat{\Gamma}_{a}^{\mu} \equiv\left(\begin{array}{cc}
\gamma^{\mu} T_{a} & 0 \\
0 & -\gamma^{\mu} T_{a}^{T}
\end{array}\right)
$$

We now transform all fields into energy-momentum space. The partition function for quarks becomes

$$
Z_{q}[A]=\int[D \bar{\Psi}][D \Psi] \exp \left[\frac{1}{2} \bar{\Psi}\left(G_{0}^{-1}+g A\right) \Psi\right] .
$$

Here, we employ a compact matrix notation,

$$
\bar{\Psi}\left(G_{0}^{-1}+g A\right) \Psi \equiv \sum_{K, Q} \bar{\Psi}(K)\left[G_{0}^{-1}(K, Q)+g A(K, Q)\right] \Psi(Q),
$$


with

$$
G_{0}^{-1}(K, Q)=\frac{1}{T}\left(\begin{array}{cc}
{\left[G_{0}^{+}\right]^{-1}(K)} & 0 \\
0 & {\left[G_{0}^{-}\right]^{-1}(K)}
\end{array}\right) \delta_{K, Q}^{(4)},
$$

where $\left[G_{0}^{ \pm}\right]^{-1}(K) \equiv \not K \pm \mu \gamma_{0}-m$, and

$$
A(K, Q) \equiv \frac{1}{\sqrt{V T^{3}}} \hat{\Gamma}_{a}^{\mu} A_{\mu}^{a}(K-Q) .
$$

The next step is to integrate out irrelevant quark modes. We define projection operators $P_{1}, P_{2}$ for relevant and irrelevant quark modes, respectively,

$$
\Psi_{1} \equiv P_{1} \Psi, \quad \Psi_{2} \equiv P_{2} \Psi, \quad \bar{\Psi}_{1} \equiv \bar{\Psi} \gamma_{0} P_{1} \gamma_{0}, \quad \bar{\Psi}_{2} \equiv \bar{\Psi} \gamma_{0} P_{2} \gamma_{0} .
$$

The Grassmann integration over the irrelevant quark fields $\bar{\Psi}_{2}, \Psi_{2}$ can be done exactly. The result for the QCD partition function is

$$
\begin{aligned}
Z & =\int[D A]\left[D \bar{\Psi}_{1}\right]\left[D \Psi_{1}\right] \exp \left\{S\left[A, \bar{\Psi}_{1}, \Psi_{1}\right]\right\} \\
S\left[A, \bar{\Psi}_{1}, \Psi_{1}\right] & \equiv S_{A}[A]+\frac{1}{2} \bar{\Psi}_{1}\left(G_{0,11}^{-1}+g B[A]\right) \Psi_{1}+\frac{1}{2} \operatorname{Tr}_{q} \ln G_{22}^{-1}[A],(15)
\end{aligned}
$$

where

$$
\begin{aligned}
g B[A] & \equiv g A_{11}-g A_{12} G_{22}[A] g A_{21}, \\
G_{22}^{-1}[A] & \equiv G_{0,22}^{-1}+g A_{22} .
\end{aligned}
$$

The quantities $G_{0,11}^{-1}, G_{0,22}^{-1}, A_{11}, A_{12}, A_{21}, A_{22}$ carry as argument a pair of quark 4-momenta, $(K, Q)$. The set of subscripts $(n m), n, m=1,2$, at these quantities indicates whether the corresponding 3-momenta $\mathbf{k}$, $\mathbf{q}$ belong to the subspace of relevant $(n=1)$ or irrelevant $(n=2)$ quark modes.

Similarly to the treatment of fermions we now define projectors $Q_{1}, Q_{2}$ for soft and hard gluon modes, respectively,

$$
A_{1} \equiv Q_{1} A, \quad A_{2} \equiv Q_{2} A .
$$

Inserting $A \equiv A_{1}+A_{2}$ into Eq. (15b), we sort the result with respect to powers of the hard gluon field, $A_{2}$,

$$
\begin{aligned}
S\left[A, \bar{\Psi}_{1}, \Psi_{1}\right] & =S\left[A_{1}, \bar{\Psi}_{1}, \Psi_{1}\right]+A_{2} J\left[A_{1}, \bar{\Psi}_{1}, \Psi_{1}\right] \\
& -\frac{1}{2} A_{2} \Delta_{22}^{-1}\left[A_{1}, \bar{\Psi}_{1}, \Psi_{1}\right] A_{2}+S_{I}\left[A_{1}, A_{2}, \bar{\Psi}_{1}, \Psi_{1}\right] .
\end{aligned}
$$

The first term in this expansion, containing no hard gluon fields at all, is simply the action (15b), with $A$ replaced by the soft gluon field $A_{1}$. The second term, $A_{2} J$, contains a single power of the hard gluon field, where $J\left[A_{1}, \bar{\Psi}_{1}, \Psi_{1}\right] \equiv J_{B}\left[A_{1}, \bar{\Psi}_{1}, \Psi_{1}\right]+J_{\text {loop }}\left[A_{1}\right]+J_{V}\left[A_{1}\right]$. The first contribution, 
$J_{B}$, arises from the coupling of the relevant fermions to the "modified" gluon field $B[A]$, i.e., from the second term in Eq. (15b). The second contribution, $J_{\text {loop }}$, arises from the term $\operatorname{Tr}_{q} \ln G_{22}^{-1}$ in Eq. (15b), plus an analogous contribution from the Fadeev-Popov determinant contained in $S_{A}[A]$. Finally, the third contribution, $J_{V}$, arises from the non-Abelian vertices in QCD.

The third term in Eq. (18) is quadratic in $A_{2}$, where $\Delta_{22}^{-1}\left[A_{1}, \bar{\Psi}_{1}, \Psi_{1}\right] \equiv$ $\Delta_{0,22}^{-1}+\Pi_{22}\left[A_{1}, \bar{\Psi}_{1}, \Psi_{1}\right]$. Here, $\Delta_{0,22}^{-1}$ is the free inverse propagator for hard gluons. Similarly to the "current" $J$ the "self-energy" $\Pi_{22}$ of hard gluons consists of three different contributions, $\Pi_{22}\left[A_{1}, \bar{\Psi}_{1}, \Psi_{1}\right]=\Pi_{B}\left[A_{1}, \bar{\Psi}_{1}, \Psi_{1}\right]+$ $\Pi_{\text {loop }}\left[A_{1}\right]+\Pi_{V}\left[A_{1}\right]$.

Finally, we collect all terms with more than two hard gluon fields $A_{2}$ in Eq. (18) in the "interaction action" for hard gluons, $S_{I}\left[A_{1}, A_{2}, \bar{\Psi}_{1}, \Psi_{1}\right]$. In order to perform the functional integration over the hard gluon fields $A_{2}$ one adds the source term $A_{2} J_{2}$ to the action (15b) and then replaces the fields $A_{2}$ in $S_{I}$ by functional differentiation with respect to $J_{2}$, at $J_{2}=$ 0 . Moving the factor $\exp \left\{S_{I}\left[A_{1}, \delta / \delta J_{2}, \bar{\Psi}_{1}, \Psi_{1}\right]\right\}$ in front of the functional $A_{2}$-integral, the latter is Gaussian and can be readily performed (after a suitable shift of $A_{2}$ ). We refrain from giving the explicit result, because we will simplify it anyway by employing the following two approximations. The first is based on the principle assumption in the construction of any effective theory, namely that soft and hard modes are well separated in momentum space. Consequently, momentum conservation does not allow a hard gluon to couple to any (finite) number of soft gluons. Under this assumption, the diagrams generated by $A_{2}\left(J_{\text {loop }}+J_{V}\right)$ will not occur in the effective theory. In the following, we shall therefore omit these terms, such that $J \equiv J_{B}$. Our second approximation is that we approximate $S_{I} \simeq 0$. With these approximations, the partition function reads

$$
Z=\int\left[D A_{1}\right]\left[D \bar{\Psi}_{1}\right]\left[D \Psi_{1}\right] \exp \left\{S_{\text {eff }}\left[A_{1}, \bar{\Psi}_{1}, \Psi_{1}\right]\right\},
$$

with the effective action

$$
\begin{aligned}
S_{\mathrm{eff}}\left[A_{1}, \bar{\Psi}_{1}, \Psi_{1}\right] & \equiv S_{A}\left[A_{1}\right]+\frac{1}{2} \bar{\Psi}_{1}\left\{G_{0,11}^{-1}+g B\left[A_{1}\right]\right\} \Psi_{1} \\
& +\frac{1}{2} \operatorname{Tr}_{q} \ln G_{22}^{-1}\left[A_{1}\right]-\frac{1}{2} \operatorname{Tr}_{g} \ln \Delta_{22}^{-1}\left[A_{1}, \bar{\Psi}_{1}, \Psi_{1}\right] \\
& +\frac{1}{2} J_{B}\left[A_{1}, \bar{\Psi}_{1}, \Psi_{1}\right] \Delta_{22}\left[A_{1}, \bar{\Psi}_{1}, \Psi_{1}\right] J_{B}\left[A_{1}, \bar{\Psi}_{1}, \Psi_{1}\right] .(20)
\end{aligned}
$$

This is the desired action for the effective theory describing the interaction of relevant quark modes, $\bar{\Psi}_{1}, \Psi_{1}$, and soft gluons, $A_{1}$. The vertices arising 
in the effective action (20) are displayed in Figs. $1-5$.

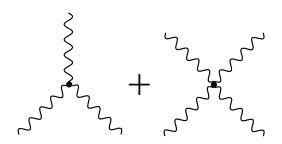

Figure 1. The three- and four-gluon vertices in $S_{A}\left[A_{1}\right]$.

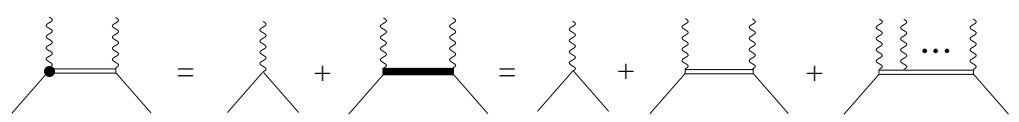

Figure 2. The term $\bar{\Psi}_{1} g B\left[A_{1}\right] \Psi_{1}$ in the effective action (20). The left-hand side is a graphical definition for this term. The right-hand side is obtained from the definition of $g B\left[A_{1}\right]$, Eq. (16a), and subsequently expanding the full propagator for irrelevant quarks, $G_{22}$ (thick solid line), in this equation in powers of the soft gluon field, $A_{1}$ (wavy line), according to Eq. (16b). A double line stands for the free propagator for irrelevant quarks, $G_{0,22}$. Thin lines stand for relevant quark fields $\Psi_{1}, \bar{\Psi}_{1}$.

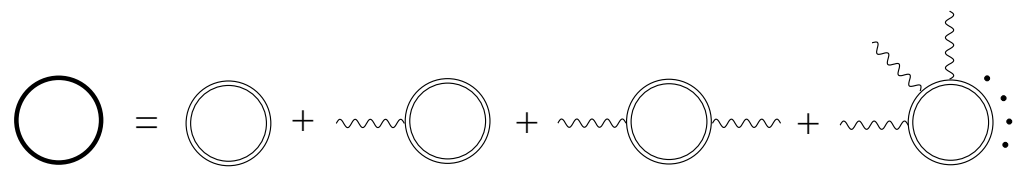

Figure 3. The term $\operatorname{Tr}_{q} \ln G_{22}^{-1}\left[A_{1}\right]$ in the effective action (20). The right-hand side is obtained upon expanding the full propagator $G_{22}$ in powers of the soft gluon field, $A_{1}$, according to Eq. (16b).

\section{Examples}

In all examples discussed in this section, the gluon projectors are chosen as

$$
\begin{aligned}
& Q_{1}\left(P_{1}, P_{2}\right) \equiv \Theta\left(\Lambda_{g}-p_{1}\right) \delta_{P_{1}, P_{2}}^{(4)}, \\
& Q_{2}\left(P_{1}, P_{2}\right) \equiv \Theta\left(p_{1}-\Lambda_{g}\right) \delta_{P_{1}, P_{2}}^{(4)} .
\end{aligned}
$$

The gluon cut-off momentum $\Lambda_{g}$ separates soft gluon fields, $A_{1} \equiv Q_{1} A$, from hard gluon fields, $A_{2} \equiv Q_{2} A$. 


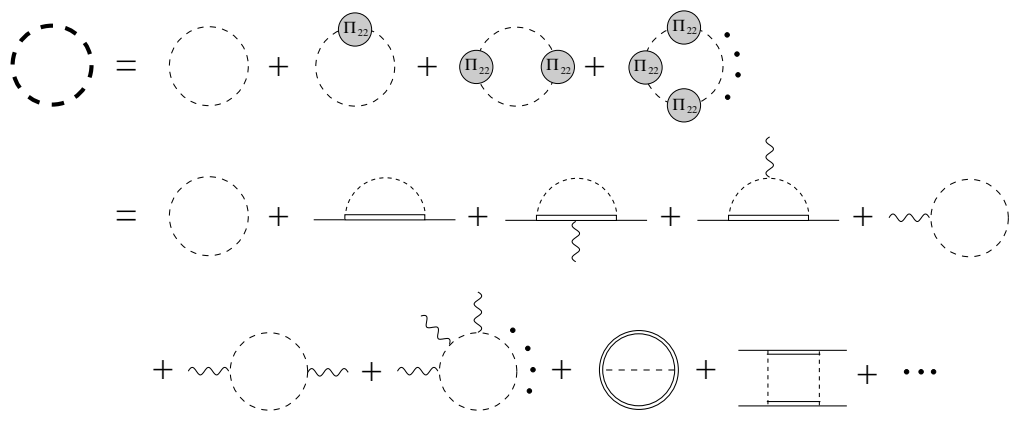

Figure 4. The term $\operatorname{Tr}_{g} \ln \Delta_{22}^{-1}\left[A_{1}, \bar{\Psi}_{1}, \Psi_{1}\right]$ in the effective action (20). The first line is obtained from the expression for $\Delta_{22}$; a dashed line denotes the free propagator for hard gluons $\Delta_{0,22}$, a bubble denotes the full self-energy $\Pi_{22}$. The second and third lines contain some examples for diagrams generated when explicitly inserting $\Pi_{22}$.

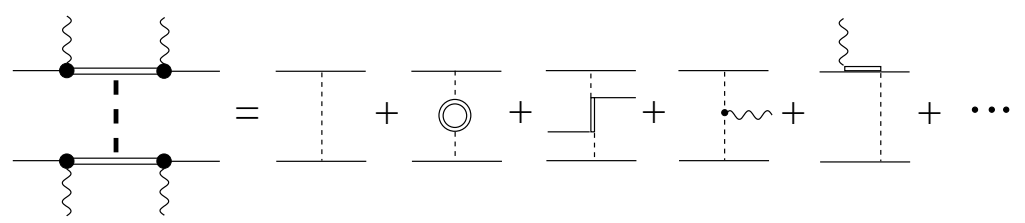

Figure 5. The term $J_{B} \Delta_{22} J_{B}$ in the effective action (20). The left-hand side serves as a graphical definition for this term, the right-hand side shows some examples for diagrams when explicitly inserting the expressions for $J_{B}$ and $\Delta_{22}$.

\subsection{HTL/HDL Effective Action}

This action defines an effective theory for massless quarks and gluons with small momenta in a system at high temperature $T$ (HTL), or large chemical potential $\mu$ (HDL). Consequently, the projectors $P_{1,2}$ for quarks are given by

$$
\begin{aligned}
& P_{1}(K, Q)=\Theta\left(\Lambda_{q}-k\right) \delta_{K, Q}^{(4)}, \\
& P_{2}(K, Q)=\Theta\left(k-\Lambda_{q}\right) \delta_{K, Q}^{(4)},
\end{aligned}
$$

The essential assumption to derive the HTL/HDL effective action is that there is a single momentum scale, $\Lambda_{q}=\Lambda_{g} \equiv \Lambda$, which separates hard modes with momenta $\sim T$, or $\sim \mu$, from soft modes with momenta $\sim g T$, or $\sim g \mu$. In order to show that the HTL/HDL effective action is contained in the effective action (20), we first note that a soft particle cannot become hard by interacting with another soft particle. This has the consequence that a soft quark cannot turn into a hard one by soft-gluon scattering. 
Therefore,

$$
g B\left[A_{1}\right] \equiv g A_{11} .
$$

Another consequence is that the last term in Eq. (20), $J_{B} \Delta_{22} J_{B}$, vanishes since $J_{B}$ is identical to a vertex between a soft quark and a hard gluon, which is kinematically forbidden. The resulting action then reads

$$
\begin{aligned}
S_{\mathrm{high} T / \mu}\left[A_{1}, \bar{\Psi}_{1}, \Psi_{1}\right] & \equiv S_{A}\left[A_{1}\right]+\frac{1}{2} \bar{\Psi}_{1}\left(G_{0,11}^{-1}+g A_{11}\right) \Psi_{1} \\
& +\frac{1}{2} \operatorname{Tr}_{q} \ln G_{22}^{-1}\left[A_{1}\right]-\frac{1}{2} \operatorname{Tr}_{g} \ln \Delta_{22}^{-1}\left[A_{1}, \bar{\Psi}_{1}, \Psi_{1}\right] .(24)
\end{aligned}
$$

We realize that the term $\operatorname{Tr}_{q} \ln G_{22}^{-1}$ generates all one-loop diagrams, where $n$ soft gluon legs are attached to a hard quark loop. This is precisely the quark-loop contribution to the HTL/HDL effective action.

Now consider the last term, $\operatorname{Tr}_{g} \ln \Delta_{22}^{-1}$. For hard gluons with momentum $\sim T$ or $\sim \mu$, the free inverse gluon propagator is $\Delta_{0,22}^{-1} \sim T^{2}$ or $\sim \mu^{2}$, while the contribution $\Pi_{\text {loop }}$ to the hard gluon "self-energy" $\Pi_{22}$ is at most of the order $\sim g^{2} T^{2}$ or $\sim g^{2} \mu^{2}$. Consequently, $\Pi_{\text {loop }}$ can be neglected and $\Pi_{22}$ only contains tree-level diagrams, $\Pi_{22} \equiv \Pi_{B}+\Pi_{V}$. Now expand $\operatorname{Tr}_{g} \ln \Delta_{22}^{-1}$ in powers of $\Pi_{22}$. The terms which contain only insertions of $\Pi_{V}$ correspond to one-loop diagrams where $n$ soft gluon legs are attached to a hard gluon loop. This is precisely the pure gluon loop contribution to the HTL effective action. For the HDL effective action, this contribution is $\sim g^{2} T^{2}$, and thus negligible.

The "self-energy" $\Pi_{B}$ contains only two soft quark legs attached to a hard quark propagator (via emission and absorption of hard gluons). Consequently, in the expansion of $\operatorname{Tr}_{g} \ln \Delta_{22}^{-1}$, the terms which contain insertions of $\Pi_{V}$ and $\Pi_{B}$ correspond to one-loop diagrams where an arbitrary number of soft quark and gluon legs is attached to the loop. It was shown in Ref. 15 that of these diagrams, only the ones with two soft quark legs and no four-gluon vertices are kinematically important and thus contribute to the HTL/HDL effective action. We have thus shown that the latter is contained in the effective action (24), and constitutes its leading contribution. 


\subsection{High-density Effective Theory}

In an effective theory for cold, dense quark matter the projectors are chosen as

$$
\begin{aligned}
& P_{1}(K, Q) \equiv\left(\begin{array}{cc}
\Lambda_{\mathbf{k}}^{+} & 0 \\
0 & \Lambda_{\mathbf{k}}^{-}
\end{array}\right) \Theta\left(\Lambda_{q}-\left|k-k_{F}\right|\right) \delta_{K, Q}^{(4)} \\
& P_{2}(K, Q) \equiv\left[\left(\begin{array}{cc}
\Lambda_{\mathbf{k}}^{-} & 0 \\
0 & \Lambda_{\mathbf{k}}^{+}
\end{array}\right)+\left(\begin{array}{cc}
\Lambda_{\mathbf{k}}^{+} & 0 \\
0 & \Lambda_{\mathbf{k}}^{-}
\end{array}\right) \Theta\left(\left|k-k_{F}\right|-\Lambda_{q}\right)\right] \delta_{K, Q}^{(4)} \cdot
\end{aligned}
$$

Here, $\Lambda_{\mathbf{k}}^{e} \equiv\left[E_{\mathbf{k}}+e \gamma_{0}(\boldsymbol{\gamma} \cdot \mathbf{k}+m)\right] /\left(2 E_{\mathbf{k}}\right)$ are projection operators onto states with positive $(e=+)$ or negative $(e=-)$ energy, where $E_{\mathbf{k}}=$ $\sqrt{\mathbf{k}^{2}+m^{2}}$ is the relativistic single-particle energy. The momentum cut-off $\Lambda_{q}$ controls how many quark modes (with positive energy) are integrated out. Thus, all quark modes within a layer of width $2 \Lambda_{q}$ around the Fermi surface are considered as relevant, while all antiquark modes and quark modes outside this layer are considered as irrelevant, cf. Fig. 6. It can be shown ${ }^{14}$ that the effective theory with the projection operators (25) is equivalent to the high-density effective theory proposed by Hong ${ }^{8,9}$ and refined by others ${ }^{10,11,12,13}$.

Armed with the effective action (20), one can compute the colorsuperconducting gap parameter in weak coupling ${ }^{14}$. One first derives the 2PI effective action ${ }^{16}, \Gamma$, with Eq. (20) as the underlying tree-level action. The stationary points of $\Gamma$ yield Dyson-Schwinger equations for the propagators of relevant quarks and soft gluons, which can be solved in a particular many-body approximation scheme. The gap equation for the color-superconducting gap parameter is equivalent to the equation for the off-diagonal component of the quark propagator in the Nambu-Gor'kov basis. The introduction of the quark and gluon cut-offs $\Lambda_{q}$ and $\Lambda_{g}$ allows for a rigorous power-counting of various contributions to the gap equation. It turns out that, in order to obtain the subleading-order result (1), one has to assume $\Lambda_{q} \lesssim g \mu \ll \Lambda_{g} \lesssim \mu$.

The situation is illustrated in Fig. 6. Relevant quark modes lie in a narrow "shell" of width $2 \Lambda_{q}$ around the Fermi surface, irrelevant quarks lie outside this shell. The leading contribution to the QCD gap equation arises from soft magnetic gluon exchange, which mediates between quark states inside a "patch" of diameter $2 \Lambda_{g}$ located on this shell. Subleading contributions arise from soft electric gluon exchange, as well as from hard magnetic and electric gluon exchange. The latter mediates between quark states in- and outside this patch, inside the shell of relevant quark modes. 


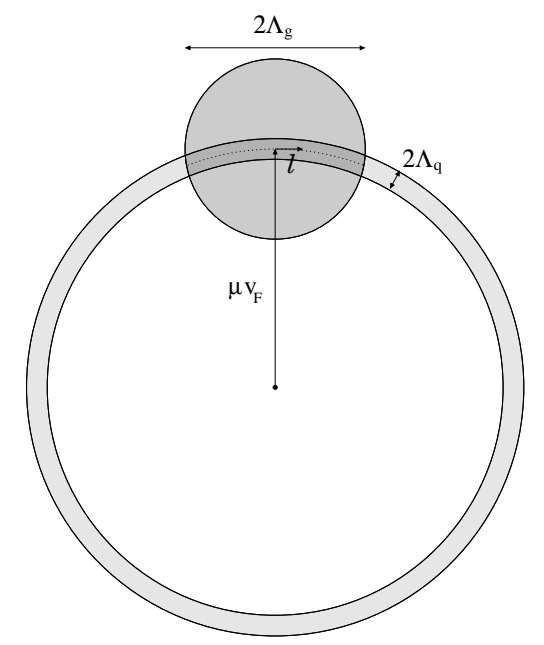

Figure 6. The shell of relevant quark modes of width $2 \Lambda_{q}$ around the Fermi surface, and the patch on this shell, characterizing the range of soft gluon interactions.

\section{Acknowledgments}

The work of Q.W. is supported by the Virtual Institute VH-VI-041 of the Helmholtz Association.

\section{References}

1. D. Bailin and A. Love, Phys. Rept. 107, 325 (1984).

2. D.T. Son, Phys. Rev. D59, 094019 (1999).

3. T. Schäfer and F. Wilczek, Phys. Rev. D60, 114033 (1999).

4. R.D. Pisarski and D.H. Rischke, Phys. Rev. D61, 051501, 074017 (2000).

5. D.K. Hong, V.A. Miransky, I.A. Shovkovy, and L.C.R. Wijewardhana, Phys. Rev. D61, 056001 (2000) [Erratum-ibid. D62, 059903 (2000)].

6. W.E. Brown, J.T. Liu, and H.c. Ren, Phys. Rev. D61, 114012 (2000); ibid. 62, 054013, 054016 (2000).

7. Q. Wang and D.H. Rischke, Phys. Rev. D65, 054005 (2002).

8. D.K. Hong, Phys. Lett. B473, 118 (2000); Nucl. Phys. B582, 451 (2000).

9. D.K. Hong, Prog. Theor. Phys. Suppl. 153, 241 (2004).

10. T. Schäfer, Nucl. Phys. A728, 251 (2003); eConf C030614, 038 (2003).

11. T. Schäfer, arXiv:hep-ph/0402032.

12. T. Schäfer and K. Schwenzer, arXiv:hep-ph/0405053.

13. G. Nardulli, Riv. Nuovo Cim. 25N3, 1 (2002).

14. P.T. Reuter, Q. Wang, and D.H. Rischke, arXiv:nucl-th/0405079.

15. E. Braaten and R.D. Pisarski, Nucl. Phys. B337, 569 (1990).

16. J.M. Cornwall, R. Jackiw, and E. Tomboulis, Phys. Rev. D10, 2428 (1974). 